\title{
Silica-Polypropylene Nanocomposites for Film Capacitors: Structure-Property Studies and the Role of Biaxial Stretching Conditions
}

\author{
I. Rytöluoto ${ }^{1,2}$, M. Niittymäki ${ }^{2}$, K. Lahti ${ }^{2}$, E. Saarimäki ${ }^{1}$, T. Flyktman $^{1}$, M. Paajanen ${ }^{1} \&$ M. Karttunen ${ }^{1}$ \\ ${ }^{1}$ VTT Technical Research Centre of Finland (VTT), Tampere, Finland \\ ${ }^{2}$ Tampere University (TAU), Electrical Engineering, Tampere, Finland \\ ilkka.rytoluoto@vtt.fi
}

\begin{abstract}
Biaxial-stretching-induced morphology development and dielectric properties of hydrophobic fumed silica-BOPP nanocomposite films were investigated. The precursor (cast) film morphology, pre-heating as well as biaxial stretching temperature and stretch ratio were found to profoundly affect the silica-BOPP film bulk morphology, surface texture and the formation of particle/agglomerate-induced cavitation upon biaxial stretching. Isothermal high-field conductivity and thermally stimulated depolarization current measurements indicated decreasing high-field/high-temperature conductivity and modification of trap density of states upon incorporation of nanosilica in comparison to neat BOPP. Large-area DC dielectric strength is, however, sensitive to thermal/mechanical stresses upon biaxial stretching, hence necessitating careful optimization of the nanocomposite formulation and processing.
\end{abstract}

\section{Introduction}

Next-generation biaxially oriented polypropylene (BOPP)silica nanocomposite (NC) films are currently being developed in the European project GRIDABLE with the aim towards applications in metallized film capacitors for e.g. voltage source converters (VSC). Our previous studies indicate that incorporation of low amounts of surface-functionalized silica nanoparticles (NPs) in a BOPP matrix provides an effective set of tools for tailoring the trap density of states, hence leading to potential improvements in insulation performance and lifetime under high field and temperature stresses [1]-[3]. However, especially for very thin films ( $5 \mu \mathrm{m}$ or less), avoiding structural inhomogeneities caused by e.g. NP agglomeration and cavitation/void formation during biaxial stretching is imperative for ensuring high dielectric performance [4]. This necessitates careful optimization of the NC formulation, compounding, film extrusion and biaxial stretching.

This paper explores our recent structure-dielectric property studies of silica-BOPP NC films, with a particular focus on understanding and controlling the biaxial-stretching-induced morphology development and the associated effects on NC charge trapping properties. For this purpose, we analyzed pilotscale silica-BOPP NC films manufactured under varied biaxial stretching conditions (pre-heating time, stretching temperature, stretch ratio). Experimental results from several measurements including optical (OM) and scanning electron microscopy
(SEM), differential scanning calorimetry (DSC), thermally stimulated depolarization current (TSDC) and DC dielectric strength are presented. The importance of optimized compounding environment as well as the degree to which the surface texture, bulk morphology and dielectric performance can be controlled by the biaxial stretching process are demonstrated, highlighting the essential role of the above factors to reach the full potential of this technology for film capacitor applications.

\section{Experimental}

\subsection{Materials and film processing}

Compounds comprising of a capacitor-grade isotactic polypropylene (PP) homopolymer matrix and $0-4.5 \mathrm{wt}-\%$ of hydrophobic fumed silica nanoparticles (NPs) were manufactured at VTT's Polymer Pilot. Dried materials were compounded using a KraussMaffei Berstorff ZE $25 \times 49$ D UTX twin-screw compounder. The screw speed was $275 \mathrm{rpm}$, temperature profile was $190-215{ }^{\circ} \mathrm{C}$, output was $15 \mathrm{~kg} / \mathrm{h}$, and nitrogen gas purge and a screen pack were utilized. The compounded strands were cooled in filtered water bath, pelletized and dried before cast film extrusion. The compounds were extruded into $\sim 350-400 \mu \mathrm{m}$ thick cast films using a Brabender Plasticorder single-screw extruder and a calendaring system inside a mobile soft-wall clean room. An additional 1.0 wt-\% PP-silica compound was also prepared by diluting a 4.5 wt- $\%$ silica-PP masterbatch during the cast film extrusion. Biaxial stretching of $10 \mathrm{~cm} \times 10 \mathrm{~cm}$ cast film specimens was performed inside a semi-clean room using a Brückner KARO IV laboratory film stretcher, with the resulting film thicknesses being in the $\sim 5-12 \mu \mathrm{m}$ range. The biaxial stretching process includes a pre-heating step followed by simultaneous biaxial stretching inside an oven: the pre-heating time and temperature as well as the biaxial stretching temperature and stretch ratio were varied in this study.

\subsection{Material characterization}

Differential scanning calorimetry (DSC) measurements were performed for $\sim 5-8 \mathrm{mg}$ disc-shaped cast film specimens encapsulated in aluminium pans using a TA Instruments Q2000 DSC in the $-50{ }^{\circ} \mathrm{C}$ to $230{ }^{\circ} \mathrm{C}$ temperature range (dynamic heating rate of $10^{\circ} \mathrm{C} / \mathrm{min}$, constant nitrogen gas purge of 50 $\mathrm{ml} / \mathrm{min}$ ). Optical microscope (OM) analysis was carried out by using a Meiji Techno ML8530 microscope equipped with a polarizing facility and a digital camera. SEM analysis was 
performed on film specimens broken in liquid nitrogen by using a JEOL JSM-6360LV scanning electron microscope. 3D surface texture analysis was carried out by using a Veeco Wyko® NT1100 optical profiling system in vertical scanning interferometry (VSI) mode from both film sides. The surface topography data was levelled by $2^{\text {nd }}$ order polynomial background subtraction and surface roughness statistics were calculated using the open-source Gwyddion software.

\subsection{Dielectric characterization}

Circular electrodes comprising of $10 \mathrm{~nm} \mathrm{Ni}$ bonding layer and $100 \mathrm{~nm}$ Au main layer were deposited on both sides of the thin film specimens using a custom-built e-beam evaporator (Instrumentti Mattila) inside an ISO 14644-1 class 6 clean room facility. High vacuum (pressure $<1 \times 10^{-6} \mathrm{mbar}$ ) and low deposition rate $(0.05-0.20 \mathrm{~nm} / \mathrm{s})$ were maintained during the evaporation process to minimize thermal and radiative damaging of the sample film. The evaporated samples were short-circuited and stored in a vacuumed desiccator prior to electrical measurements.

Isothermal charging current and TSDC measurements were performed using a liquid $\mathrm{N}_{2}$-based temperature control system with an accuracy of $\pm 0.1{ }^{\circ} \mathrm{C}$ (Novocontrol Novocool), a high voltage DC source (Keithley 2290E-5) and an electrometer (Keithley 6517B). A shielded sample cell equipped with a PT100 temperature sensor (Novocontrol BDS1200 HV sample cell) and shielded cables were used. A diode-based overload protection circuit and a $100 \mathrm{k} \Omega$ series resistor were utilized to protect the electrometer in case of a sample breakdown during high voltage application. Contact with the metallized sample film was made using conductive fluorosilicone rubber. The isothermal charging current and TSDC measurement procedure was: (i) polarization at DC field $E_{p}=100 \mathrm{~V} / \mu \mathrm{m}$ for $40 \mathrm{~min}$ under isothermal conditions at $T_{p}=80^{\circ} \mathrm{C}$, (ii) rapid cooling to $T_{0}=$ $-50{ }^{\circ} \mathrm{C}$; hold isothermally for $5 \mathrm{~min}$, (iii) removal of the poling voltage and short-circuiting of the sample; hold isothermally for 3 min, (iv) linear heating at $\beta=3.0^{\circ} \mathrm{C} / \mathrm{min}$ up to $T_{\max }=\sim 140^{\circ} \mathrm{C}$. The polarization/depolarization current was measured for the whole duration of the above process.

Room temperature large-area DC breakdown characteristics of the biaxially stretched films were measured using a selfhealing breakdown method detailed in [5], [6]. Test capacitors with active area of $81 \mathrm{~cm}^{2}$ were assembled by sandwiching the sample film between two sheets of commercial metallized BOPP film, the metallized surfaces of which were facing towards the sample film. Self-healing breakdown measurements were performed in atmospheric air using a slow-rate-of-rise voltage ramp profile defined in the IEC-60243-1 standard. Analysis of the multi-breakdown data sets was carried out in MATLAB and statistical analysis was based on Weibull statistics.

\section{Results and discussion}

\subsection{Morphology formation upon biaxial stretching}

\section{1) Initial cast film morphology and the effect of pre-heating}

It is well known that the initial cast film morphology plays a significant role in the morphology formation upon biaxial stretching [6]. Mixed type $\alpha / \beta$-form crystalline structure was confirmed by DSC analysis for each cast film, as is exemplified in Figure 1 for a 4.5 wt- $\%$ silica-PP and unfilled reference PP compounds. The $1^{\text {st }}$ melting endotherms showed predominantly monoclinic $\alpha$-form crystalline structure (peak melting temperature of $\sim 163{ }^{\circ} \mathrm{C}$ ), with only faint traces of hexagonal $\beta$ form crystallinity (observable as double $\beta$-melting peaks at $\sim 140$ ${ }^{\circ} \mathrm{C}$ and $\sim 147{ }^{\circ} \mathrm{C}$ [7]) being visible. For brevity, the effect of silica on PP morphology is not discussed here. To better understand the morphological changes taking place during the pre-heating stage before biaxial stretching of the cast film, several cast film specimens were treated by exposing them to the pre-heating conditions inside the KARO IV equipment without subsequently stretching the films. After the heat treatment the cast films were taken out from the oven and let to cool down under room temperature conditions. DSC analysis of the above-treated cast films (Figure 1, top curves) indicated: (i) transformation of the $\beta$-form crystallites into $\alpha$-form, (ii) lamellar thickening of the unmolten $\alpha$-form crystallites and (iii) increase in degree of crystallinity. The above morphological changes became more prominent with increasing temperature, and can play a significant role for the morphology formation upon biaxial stretching [8], as will be discussed below.

\section{2) Morphological characteristics of the silica-BOPP films}

Figure 4a presents an exemplifying OM micrograph of a 4.5 wt- $\%$ silica-BOPP film biaxially stretched at $157^{\circ} \mathrm{C}$, from which several prominent features for the biaxially stretched films can be observed. Firstly, the dark circular regions seen in the OM micrographs correspond to the crater-like surface texture of the film, a typical feature for BOPP films [9]. A more striking feature in Figure 4a is, however, the presence of "island-like" light-reflecting regions which can be attributed to porosity and/or voids along the stretching direction inside the BOPP film [6]. This is an atypical and unwanted feature for capacitor films. Figure $4 \mathrm{~b}$ and Figure $4 \mathrm{c}$ respectively show a higher magnification of one such void under reflected and transmitted light: particles are clearly seen in the centre of the void (especially in transmission mode), with this indicating that the

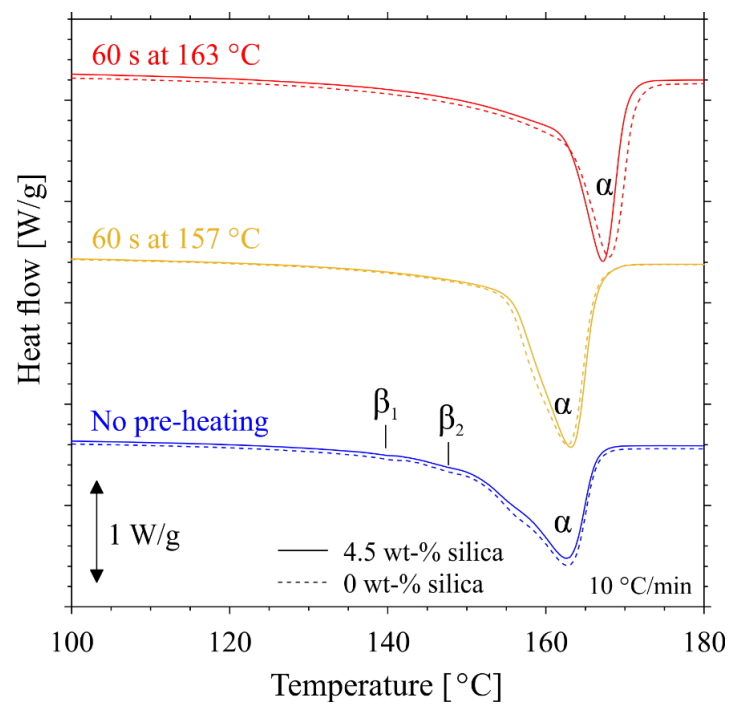

Figure 1. DSC $1^{\text {st }}$ heating scans of $4.5 \mathrm{wt}-\%$ silica-PP (solid) and unfilled PP (dashed) cast films (before and after pre-heating). The shift of $\alpha$ - melting peak to higher temperature and the decrease of $\alpha$ - peak width indicates $\alpha$-form lamellar thickening and perfection. 

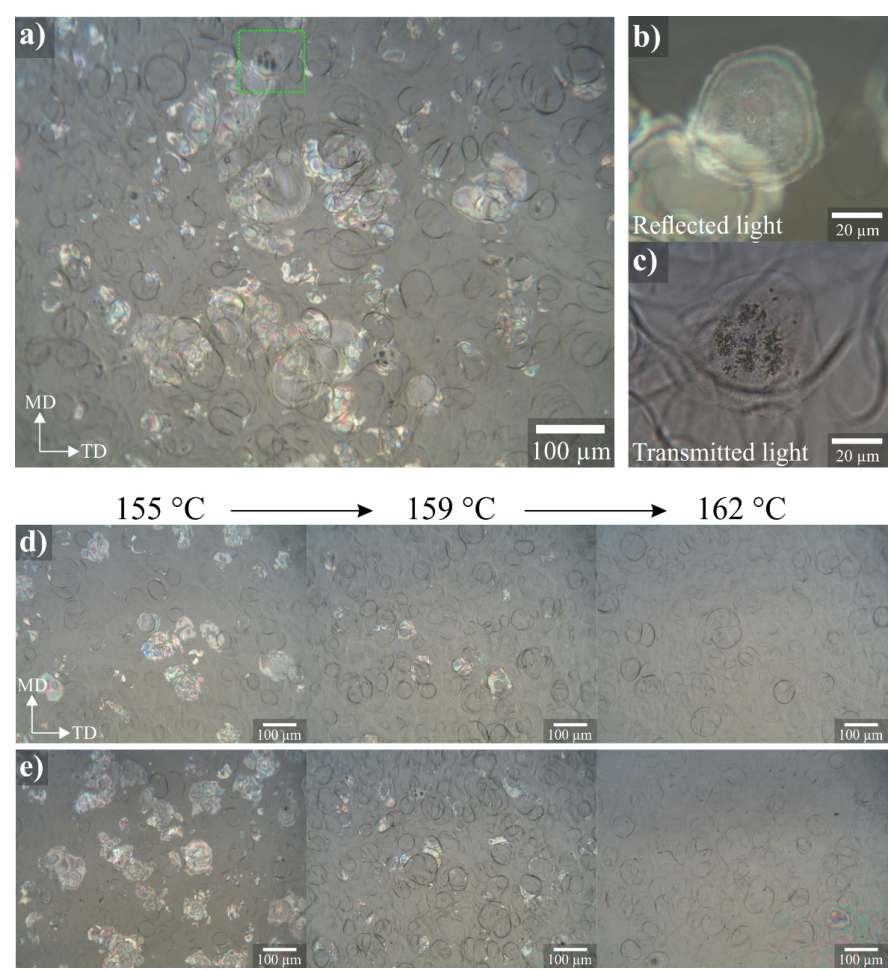

Figure 4. a) $\mathrm{OM}$ image of a $4.5 \mathrm{wt}-\%$ silica-BOPP film (stretching temperature $157^{\circ} \mathrm{C}$, stretch ratio $6.0 \times 6.0$ ). A higher magnification of the circled (green) area is shown in b) under reflected light and in $\mathbf{c}$ ) under transmitted light. The influence of increasing pre-heating and stretching temperature on the film morphology is presented in d) and e) for 4.5 wt- $\%$ silica-BOPP and unfilled silica-BOPP films, respectively. MD: machine direction, TD: transverse direction.

void formation mechanism upon biaxial stretching may be particle-induced. While the observed particles are presumably silica agglomerates [2], external particles (such as burnt polymer residue) originating from the compounding equipment or surrounding environment cannot be ruled out at this point. Interestingly, similar voids were also observed in the unfilled reference films (Figure 4e), with this pointing towards the particles comprising not only of silica. The presence of voids was later confirmed by SEM cross-sectional imaging of specimens broken in liquid nitrogen (not shown).

\section{3) Biaxial stretching parameters vs. film morphology}

The influence of biaxial stretching temperature (from $155^{\circ} \mathrm{C}$ to $163{ }^{\circ} \mathrm{C}$ ) and stretch-ratio (up to $6.0 \times 6.0$ ) on the film morphology was studied in more detail. OM analysis of the morphological evolution of the studied silica-BOPP films under various biaxial stretching temperatures is presented in Figure $4 \mathrm{~d}$ and Figure $4 \mathrm{e}$ for a $4.5 \mathrm{wt}-\%$ silica composite and unfilled reference, respectively. Surface roughness characteristics measured from the both sides of the films using optical profilometry are presented in Figure 2. Finally, a comparison of machine directional (MD) stretch forces vs. stretch ratio at different temperatures is presented in Figure 3 for a $4.5 \mathrm{wt}-\%$ silica-BOPP composite.

At relatively low stretching temperatures $\left(155^{\circ} \mathrm{C}\right)$ voids were clearly observable using OM imaging (Figure $4 \mathrm{~d}-\mathrm{e}$ ) and the stretching forces (Figure 3) exhibited a typical ductile deformation behavior, showing a yield region around 1.3 stretch

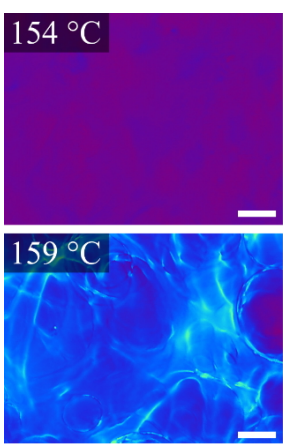

a)
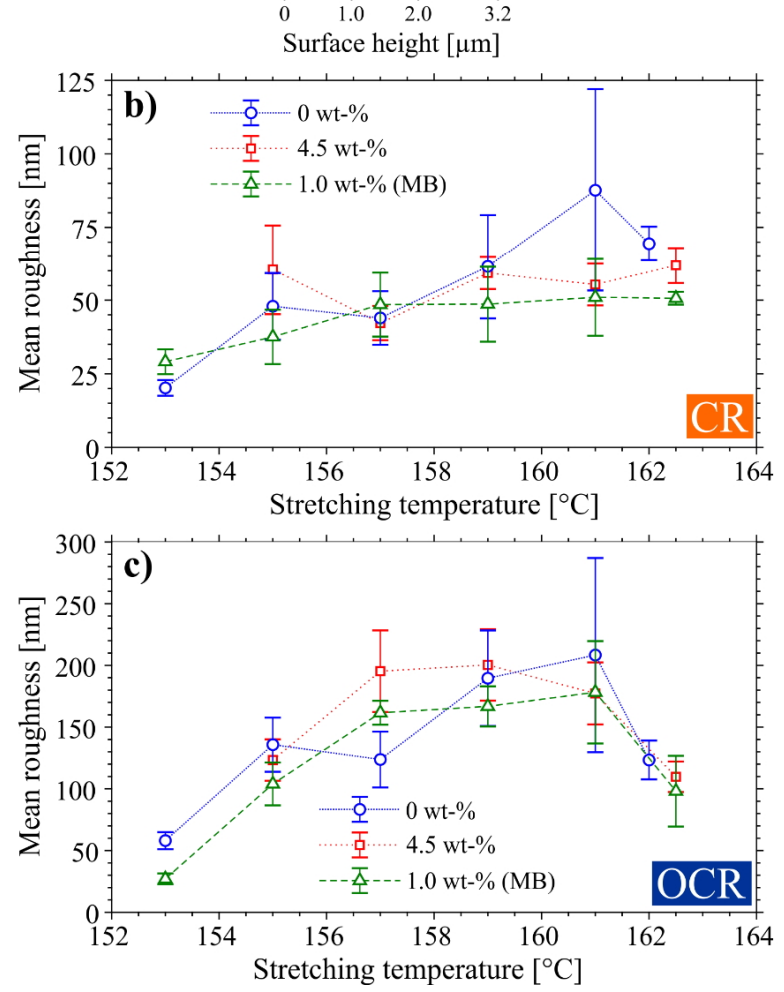

Figure 2. a) Surface height profiles of $4.5 \mathrm{wt}-\%$ silica-BOPP film at various biaxial stretching temperatures. The scale bar is $100 \mu \mathrm{m}$. b) and c) show the mean surface roughness as a function of stretching temperature for the chill-roll side (CR) and opposite side of chill-roll (OCR), respectively. The error bars denote standard deviation.

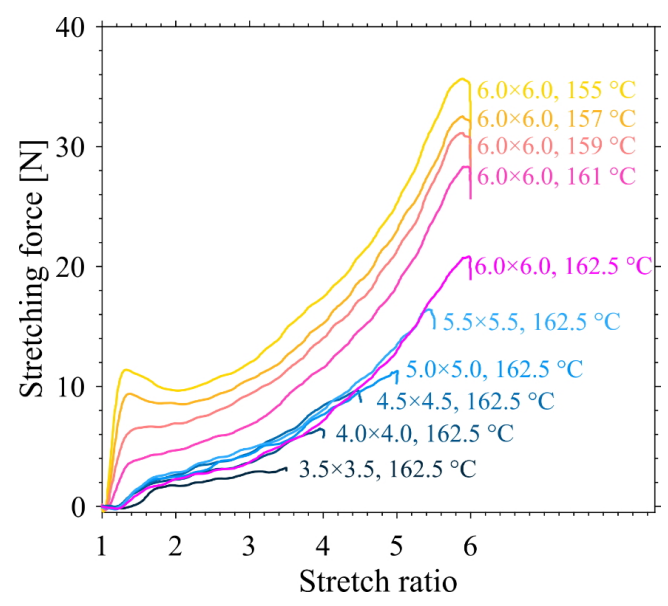

Figure 3. Force vs. stretch ratio at different stretch temperatures and elongations (4.5 wt-\% silica). For clarity, only MD-directional forces are shown (TD forces are similar under equibiaxial stretching). 
ratio followed by neck propagation and strain hardening, being consistent with e.g. Capt [8]. As the stretching temperature was increased from $155^{\circ} \mathrm{C}$, the voids observable using $\mathrm{OM}$ were found to gradually decrease in size and quantity until at $162{ }^{\circ} \mathrm{C}$ (close to the melting point of the polymer), they were eliminated completely (Figure 4d-e). With increasing stretching temperature the stretching forces decreased and began to show rubber-like deformation characteristics with no observable yield point (Figure 3). Simultaneously, the BOPP film surface roughness (Figure 2) was found to exhibit an increasing trend with the stretching temperature. The formation of crater-like surface roughness upon biaxial stretching is closely related with the spherulitic morphology and collapse of trans-crystalline surface layer in the precursor cast film [6], [10]. The increase in surface roughness with the stretching temperature is in line with Tamura et al. [10], and may be related with higher efficiency of the stretching-induced collapse of the crystal grain layer within the surface layers of the film.

\section{4) On the void formation mechanism}

The obtained results indicate that the biaxial stretching temperature is closely related with the presence or absence of voids inside the films. We note two mechanisms which may play a role here. Firstly, following the model proposed by Qaiss et al. [11], upon biaxial stretching the local stress concentration near the surface of the agglomerates/particles can result in decohesion at the particle-matrix interface, with this resulting in the initial pore formation and subsequent development into larger voids. The size of the particles/agglomerates and the stretching conditions obviously have a significant effect in the above process [12]. When the biaxial stretching temperature is increased close to the melting point of the polymer, either the voided structure collapses [11], or the movement of the polymer chains and particles along the stretching direction becomes easier [12], hence eliminating the void formation. Indeed, the amount of molten polymer just above its melting point bearing high molecular mobility and nucleating power ("structured melt") increases with the pre-heating/stretching temperature [8], hence reducing the stress concentration near particulates and giving rise to e.g. higher degree of crystallinity. On the other hand, a second relevant mechanism for the void formation is related to the presence of $\beta$-form crystallites in the precursor cast film: this has been previously associated with stretching-induced porosity/void formation in laboratory-scale BOPP films due to inter-( $\beta$-)lamellar cavitation and volume contraction effect due to the $\beta \rightarrow \alpha$ crystal transformation [6]. It is however interesting that the DSC analysis of the pre-heated cast films (Figure 1) indicated the absence of $\beta$-form crystallinity when biaxial stretching begins: this is understandable as the pre-heating was done above the melting point of $\beta$-crystallites $\left(>147^{\circ} \mathrm{C}\right)$. We also remark that the temperature of the calendaring system during cast extrusion was set so that the crystallization conditions for $\beta$-crystal formation were mostly unfavourable. Thus, the void formation for the films studied herein is presumably not dictated merely by the porosity formation related to $\beta$-crystallites [7], but it is rather caused by a combination of above effects. However, even though the voids are eliminated by increasing the pre-heating and biaxial stretching temperature, the agglomerates/particles still remain in the film, having an impact on the dielectric properties [13].

\subsection{Conductivity}

Significant differences in conductivity (at the end of isothermal polarization phase) were observed depending on the silica concentration and biaxial stretching parameters. Figure 5a presents the charging current vs. time $(I-t)$ for various silicaBOPP films under isothermal polarization at $80{ }^{\circ} \mathrm{C}$. The charging current exhibited a typical inverse power law relationship $I \sim t^{-n}$ with the exponent $n$ of $\sim 0.6$, similar to commercial capacitor BOPP [14]. Figure 5b-d respectively summarize the dependence of conductivity (calculated as $\sigma=J / E$, where $J$ is the current density and $E$ is the poling field) at the end of polarization as a function of silica content, stretch ratio and pre-heating/stretching temperature. As shown in Figure $5 \mathrm{~b}$, increasing silica content was found to decrease the high-field conductivity at $80{ }^{\circ} \mathrm{C}$ in comparison to neat BOPP, with this trend being consistent also for the masterbatch (MB) material diluted during cast extrusion. Compounding the silica-PP material twice before cast extrusion resulted in a further (small) reduction in conductivity. Similar trend was also observed at 100 ${ }^{\circ} \mathrm{C}$ (not presented here). These results are encouraging for film capacitor applications and indicate a modification of the charge trap structure upon incorporation of nanosilica [1], [15]. Moreover, the 4.5 wt- $\%$ silica-BOPP films also exhibited differing behavior with respect to biaxial stretch-ratio (Figure 5c) and stretching temperature (Figure 5d): while the unfilled reference exhibited increasing conductivity with increasing biaxial stretching ratio and temperature, the silica-PP material was mostly unaffected by these factors. That is, from the morphological point-of-view, the stretching-induced structural changes did not seem to affect the conductivity of the silicaBOPP films significantly while the opposite was true for the neat BOPP, despite the fact that a relatively larger amount of agglomerates and voids were seen in the silica-BOPP films.
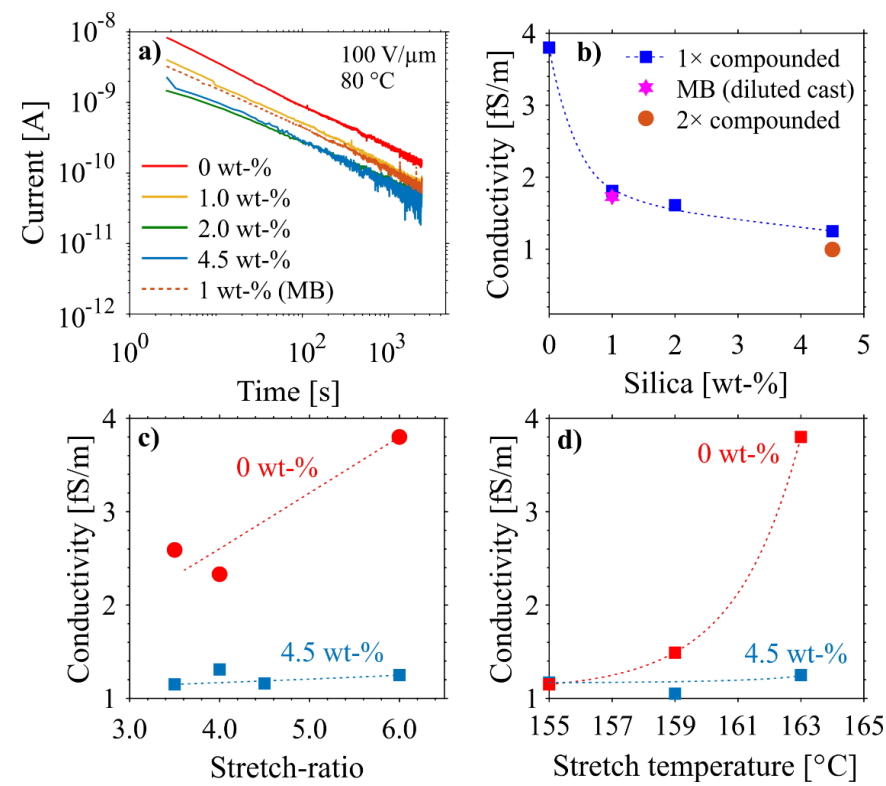

Figure 5. a) Isothermal charging current vs. time $\left(\sim 162{ }^{\circ} \mathrm{C}\right.$ stretch temperature, $6.0 \times 6.0$ stretch ratio). b) Conductivity at the end of poling as a function silica content $\left(\sim 162{ }^{\circ} \mathrm{C}\right.$ stretch temperature, $6.0 \times 6.0$ stretch ratio). c) Conductivity at the end of poling as a function stretch ratio $\left(\sim 162{ }^{\circ} \mathrm{C}\right.$ stretch temperature). d) Conductivity at the end of poling as a function pre-heating/stretch temperature $(6.0 \times 6.0$ stretch ratio). In c)-d) the dashed curves are to guide eyes. 


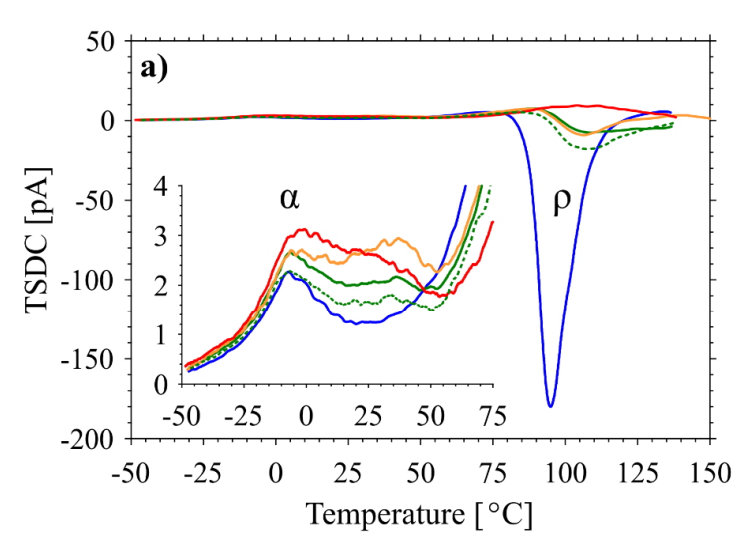

$-0 \mathrm{wt}-\%-1.0 \mathrm{wt}-\%-2.0 \mathrm{wt}-\%-4.5 \mathrm{wt}-\% \cdots \cdots \cdot 1.0 \mathrm{wt}-\%$ (MB)

\subsection{TSDC}

First, we briefly summarize the effect of nanosilica on the thermally stimulated depolarization current of BOPP. TSDC spectra of 0-4.5 wt-\% silica-BOPP films are presented in Figure 6a: the unfilled BOPP film shows (i) a small heteropolar $(\alpha-)$ relaxation peak at $-6{ }^{\circ} \mathrm{C}$ associable with detrapping of charge from shallow traps as the polymer passes the glass-transition and (ii) a strong homopolar space charge $(\rho-)$ relaxation in high temperature region $\left(\sim 100^{\circ} \mathrm{C}\right)$ which is due to the thermal release of charges from deep traps. Upon incorporation of nanosilica, the trap distribution is modified profoundly. The TSDC spectra above the glass transition temperature becomes more pronounced and complex, indicating a higher density of shallow traps in the silica-containing materials. Moreover, in the high temperature region, the silica-BOPP films exhibit greatly reduced TSDC intensity, being indicative of modification of the deep trap density with nanosilica. The results indicate a modification of trap density of states and a more homogeneous distribution of charge in the silica-containing samples. Such properties have also been verified from similar cast and biaxially oriented films in another study [1] using complementary experimental methods (PEA, conductivity and isothermal depolarization current decay).

The effects of biaxial stretch ratio and pre-heating/stretching temperature are respectively compared in Figure $6 \mathrm{~b}$ and Figure $6 \mathrm{c}$ for a $4.5 \mathrm{wt}-\%$ silica-BOPP film. Both increasing biaxial stretching ratio and temperature were found to increase the high temperature TSDC peak intensity (around $100{ }^{\circ} \mathrm{C}$ ), hence implying an increase deep trap density with the above factors. Considering the increasing biaxial stretch ratio, the above effect can be attributed with a modification of crystallinity, crystallite size and crystallite orientation [8], as these properties affect the quantity of charge trap sites at the amorphous-crystalline boundaries [16], [17]. The (asymmetrical) thickness-directional morphology "gradient" [18] and surface texture characteristics of BOPP likely play a significant role here [6]. On the other hand, the increase of deep trap density with increasing preheating/stretching temperature is somewhat unexpected, as the films biaxially stretched at lower temperatures exhibited relatively large amount of voids (see Figure 4) and were thus expected to show high charge trapping propensity, as is typically observed for voided/cellular iPP films [19]. It is thus likely that
Figure 6. a) TSDC spectra of $0-4.5$ wt- $\%$ silica-BOPP films $\left(\sim 162{ }^{\circ} \mathrm{C}\right.$ stretch temperature, $6.0 \times 6.0$ stretch ratio). The inset shows a zoomed-in portion of the "shallow trap region" from $-50{ }^{\circ} \mathrm{C}$ to $75^{\circ} \mathrm{C}$. Glass-transition $(\alpha-)$ and space charge $(\rho-)$ relaxations are labelled. b) TSDC spectra of $4.5 \mathrm{wt}-\%$ silica-BOPP films produced using various stretch ratios $\left(\sim 162{ }^{\circ} \mathrm{C}\right.$ stretch temperature). c) TSDC spectra of $4.5 \mathrm{wt}-\%$ silica-BOPP films produced using various pre-heating/stretching temperatures.

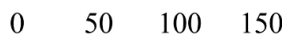

Temperature $\left[{ }^{\circ} \mathrm{C}\right]$

the quantity and dimensions of the voids even at $155^{\circ} \mathrm{C}$ were not large enough to have a significant effect on charge trapping, and hence the observed increase in deep trap density with increasing pre-heating/stretching temperature is mostly associated with other morphological changes [8].

\subsection{Large-area dielectric strength}

The effects of biaxial stretch ratio and pre-heating/stretching temperature on the room temperature large-area DC dielectric breakdown characteristics of 4.5 wt- $\%$ silica-BOPP film are summarized in Figure 7, where Weibull $\alpha$ is the scale parameter (breakdown strength at $63.2 \%$ probability) and Weibull $\beta$ is the shape parameter (i.e. distribution homogeneity). Firstly, as expected, an increase in breakdown strength (Weibull $\alpha$ ) was observed with increasing stretch ratio (Figure 7a), along with an increasing trend in Weibull $\beta$. The increase in dielectric strength with stretching can be attributed to increased chain orientation
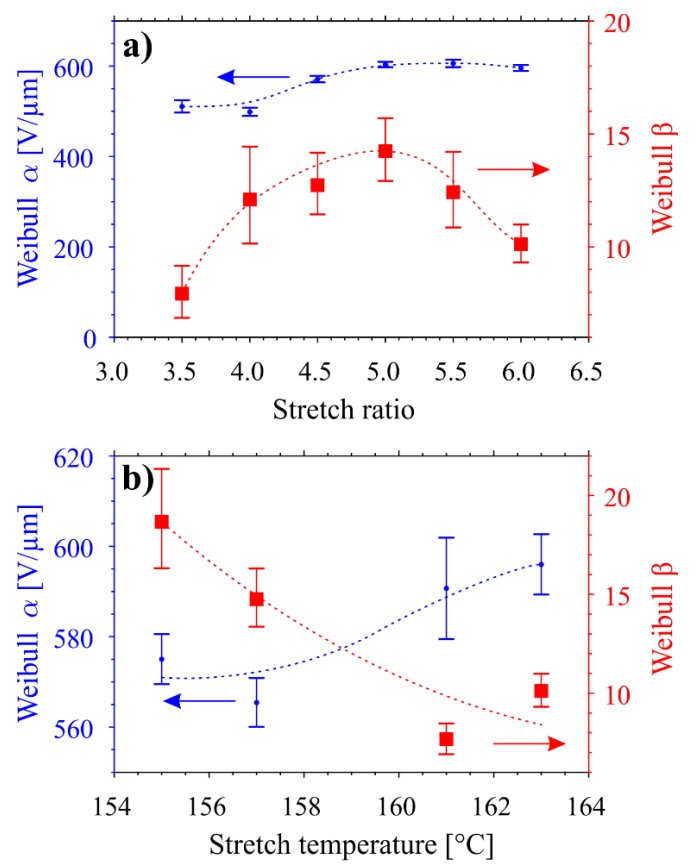

Figure 7. Weibull parameters (breakdown strength) of a 4.5 wt- $\%$ silica-BOPP film as a function of a) stretch ratio $\left(\sim 162{ }^{\circ} \mathrm{C}\right.$ stretch temperature) and b) stretch temperature $(6.0 \times 6.0$ stretch ratio). The dashed curves are to guide eyes. 
and formation of fibrillar BOPP morphology as discussed elsewhere [6], and may also be related with higher trap density (see Figure 6). On the other hand, while increasing biaxial stretching temperature also resulted in a slight increase in dielectric strength (Weibull $\alpha$ ), a significant reduction in Weibull $\beta$ was concurrently observed. Thus, biaxial stretching very close to the melting point of the polymer likely resulted in partial melting and poor orientation of the polymer chains, hence resulting in decreased breakdown performance even though the voids and cavities, which in principle may affect the dielectric strength [13], [20], were eliminated.

\section{Conclusions}

Biaxial-stretching-induced morphology development and dielectric properties of hydrophobic fumed silica-BOPP nanocomposite films were investigated. Incorporation of fumed nanosilica can bring about promising dielectric property modifications such as decreased high field/high temperature conductivity and modification of the trap density of states, however, further improvement of silica dispersion and film morphology are still seen necessary. Achieving homogeneous morphology in biaxially oriented thin films is perhaps one of the most challenging tasks in the field of dielectric nanocomposites, as issues such as nanoparticle agglomeration and structural inhomogeneities (e.g. cavities) become increasingly critical as the film thickness is decreased and the material is pushed to its limits. While it is possible to eliminate the formation of particleinduced voids and cavities during biaxial stretching by increasing the stretching temperature, this can also impact the dielectric strength of the material. These results highlight the essential role of optimizing the nanocomposite formulation, compounding and film processing to reach the full potential of this technology for film capacitor applications.

\section{Acknowledgement}

This project has received funding from the European Union's Horizon 2020 research and innovation programme under grant agreement No 720858.

\section{References}

[1] I. Rytöluoto et al., "Compounding, Structure and Dielectric Properties of Silica-BOPP Nanocomposite Films," in 2018 IEEE 2nd International Conference on Dielectrics (ICD), 2018, pp. 1-4.

[2] I. Rytöluoto, K. Lahti, M. Karttunen, M. Koponen, S. Virtanen, and M. Pettersson, "Large-area dielectric breakdown performance of polymer films - Part II: Interdependence of filler content, processing and breakdown performance in polypropylene-silica nanocomposites," IEEE Trans. Dielectr. Electr. Insul., vol. 22, no. 4, pp. 2196-2206, 2015.

[3] G. C. Montanari, P. Seri, M. Ritamaki, K. Lahti, I. Rytoluoto, and M. Paajanen, "Performance of nanoparticles in the electrical behavior of DC capacitor films," Proc. IEEE Int. Conf. Prop. Appl. Dielectr. Mater., vol. 2018-May, pp. 41-44, 2018.

[4] S. K. Kumar, B. C. Benicewicz, R. A. Vaia, and K. I. Winey, "50th Anniversary Perspective: Are Polymer Nanocomposites Practical for Applications?," Macromolecules, vol. 50, no. 3, pp. 714-731, Feb. 2017.

[5] I. Rytöluoto, K. Lahti, M. Karttunen, and M. Koponen, "Large-area dielectric breakdown performance of polymer films - Part I: Measurement method evaluation and statistical considerations on area-dependence," IEEE Trans. Dielectr. Electr. Insul., vol. 22, no. 2, pp. 689-700, 2015.

[6] I. Rytöluoto, A. Gitsas, S. Pasanen, and K. Lahti, "Effect of film structure and morphology on the dielectric breakdown characteristics of cast and biaxially oriented polypropylene films," Eur. Polym. J., vol. 95, 2017.

[7] G. T. Offord et al., "Porosity enhancement in $\beta$ nucleated isotactic polypropylene stretched films by thermal annealing," Polymer (Guildf)., vol. 54, no. 10, pp. 2577-2589, 2013.

[8] L. Capt, "Simultaneous biaxial stretching of isotactic polypropylene films in the partly molten state," McGill University, 2003.

[9] J. L. Nash, "Biaxially oriented polypropylene film in power capacitors," Polym. Eng. Sci., vol. 28, no. 13, pp. 862-870, 1988.

[10] S. Tamura, K. Ohta, and T. Kanai, "Study of crater structure formation on the surface of biaxially oriented polypropylene film," J. Appl. Polym. Sci., vol. 124, no. 4, pp. 2725-2735, May 2012.

[11] A. Qaiss, H. Saidi, O. Fassi-Fehri, and M. Bousmina, "Porosity formation by biaxial stretching in polyolefin films filled with calcium carbonate particles," J. Appl. Polym. Sci., vol. 123, no. 6, pp. 3425-3436, Mar. 2012.

[12] X. Zhang, Q. Xing, R. Li, R. Wang, and D. Wang, "Deformation investigation on $\mathrm{iPP} / \mathrm{SiO} 2$ composites: Influence of stretching temperature and particle size on morphology evolution and crystalline structure of thin films," Chinese J. Polym. Sci., vol. 31, no. 2, pp. 275-284, 2013.

[13] X. Qiu, F. Groth, W. Wirges, and R. Gerhard, "Cellular polypropylene foam films as DC voltage insulation and as piezoelectrets - A comparison," IEEE Trans. Dielectr. Electr. Insul., vol. 25, no. 3, pp. 829834, Jun. 2018.

[14] I. Rytöluoto, M. Ritamäki, and K. Lahti, "Short-term dielectric performance assessment of BOPP capacitor films: A baseline study," in 2018 12th International Conference on the Properties and Applications of Dielectric Materials (ICPADM), 2018, pp. 289-292.

[15] G. C. Montanari, D. Fabiani, F. Palmieri, D. Kaempfer, R. Thomann, and R. Mülhaupt, "Modification of electrical properties and performance of EVA and PP insulation through nanostructure by organophilic silicates," IEEE Trans. Dielectr. Electr. Insul., vol. 11, no. 5, pp. 754-762, 2004.

[16] K. Yahagi, "Dielectric Properties and Morphology in Polyethylene," IEEE Trans. Electr. Insul., vol. EI-15, no. 3, pp. 241-250, 1980.

[17] J. K. Nelson, "Breakdown Strength of Solids," in Engineering Dielectrics Volume IIA Electrical Properties of Solid Insulating Materials: Molecular Structure and Electrical Behavior, R. Bartnikas and R. Eichhorn, Eds. Philadelphia: ASTM International, 1983, pp. 445-520.

[18] A. Thielen, J. Niezette, G. Feyder, and J. Vanderschueren, “Thermally stimulated current study of space charge formation and contact effects in metal-polyethylene terephthalate film-metal systems. I. Generalities and theoretical model," J. Phys. Chem. Solids, vol. 57, no. 11, pp. 1567-1580, Nov. 1996.

[19] J. Hillenbrand, N. Behrendt, V. Altstädt, H.-W. Schmidt, and G. M. Sessler, "Electret properties of biaxially stretched polypropylene films containing various additives," J. Phys. D. Appl. Phys., vol. 39, no. 3, pp. 535-540, 2006.

[20] E. Ildstad and T. Haave, "Conduction and partial discharge activity in HVDC cable insulation of lapped polypropylene films," in ICSD'01. Proceedings of the 20001 IEEE 7th International Conference on Solid Dielectrics (Cat. No.01CH37117), 2001, pp. 137-140. 